\title{
Tumor burden and liver function in HCC patient selection for selective internal radiation therapy: SARAH post-hoc study
}

\author{
Daniel H Palmer ${ }^{\star 1}$, Neil S Hawkins², Valérie Vilgrain ${ }^{3,4}$, Helena Pereira ${ }^{5,6}$, Gilles \\ Chatellier ${ }^{5,6}$ \& Paul J Ross ${ }^{7,8}$ \\ ${ }^{1}$ Liverpool CR UK/NIHR Experimental Cancer Medicine Centre, University of Liverpool, Liverpool, L69 3BX, United Kingdom \\ ${ }^{2}$ Institute of Health \& Wellbeing, University of Glasgow, Glasgow, G12 8RZ, United Kingdom \\ ${ }^{3}$ Assistance Publique - Hôpitaux de Paris, Hôpitaux Universitaires Paris Nord Val de Seine, Hôpital Beaujon, 92118 Clichy, France \\ ${ }^{4}$ Centre de Recherche de I'Inflammation, INSERM U1149, Université Paris Sorbonne Cité, Paris, France \\ ${ }^{5}$ Assistance Publique - Hôpitaux de Paris, Hôpital européen Georges-Pompidou, Unité d'épidémiologie et de Recherche Clinique, \\ Paris, 75015 France \\ ${ }^{6}$ Module Épidémiologie Clinique, Centre d'Investigation Clinique 1418, INSERM, Paris, France \\ ${ }^{7}$ Department of Medical Oncology, Guy's \& St Thomas' NHS Foundation Trust, London, SE1 9RT, United Kingdom \\ ${ }^{8}$ Department of Oncology, King's College Hospital NHS Foundation Trust, London, SE5 9RS, United Kingdom \\ *Author for correspondence: Tel.: +0151 794 8333; daniel.palmer@liverpool.ac.uk
}

\begin{abstract}
Aim: To determine whether a liver tumor burden $\leq 25 \%$ and well-preserved liver function (albuminbilirubin grade 1) are appropriate criteria for identifying patients with unresectable hepatocellular carcinoma who may benefit from selective internal radiation therapy (SIRT) using ${ }^{90} y$ ttrium resin microspheres versus sorafenib. Patients \& methods: Post-hoc analysis of patients in the intention-to-treat population of the SARAH trial (SIRT vs sorafenib) with $\leq 25 \%$ tumor burden and albumin-bilirubin grade 1. Primary end point: overall survival. Results: Median overall survival was 21.9 months $(95 \% \mathrm{Cl}: 15.2-32.5, \mathrm{n}=37)$ with SIRT and 17.0 months (11.6-20.8, $\mathrm{n}=48$ ) with sorafenib (hazard ratios: 0.73 [95\% Cl: $0.44-1.21 ; \mathrm{p}=0.22]$ ). Conclusion: A combination of good liver function and low tumor burden may be relevant for selection of hepatocellular carcinoma patients for SIRT.
\end{abstract}

First draft submitted: 21 October 2019; Accepted for publication: 18 November 2019; Published online: 4 December 2019

Keywords: ${ }^{90}$ yttrium $\bullet$ albumin-bilirubin grade $\bullet$ hepatocellular carcinoma $\bullet$ resin microspheres $\bullet$ selective internal radiation therapy $\bullet$ SIR-spheres $\bullet$ sorafenib $\bullet$ tumor burden

The incidence of hepatocellular carcinoma (HCC) is increasing worldwide, and many patients have intermediateor advanced-stage HCC at presentation when potentially curative treatments, such as surgical resection or tumor ablation, are no longer practicable. For patients with advanced-stage disease treatment is limited to systemic therapies. First-line treatment is based on the tyrosine kinase inhibitors sorafenib and lenvatinib [1-3]. Sorafenib has been used as first-line treatment for advanced HCC following the publication of the Phase III SHARP trial, which showed an overall survival (OS) benefit of sorafenib versus placebo [1,2]. However, adverse events (AE) associated with sorafenib treatment are frequent and can necessitate discontinuation of treatment and dose reduction $[1,2,4]$. Lenvatinib is noninferior to sorafenib in the treatment of HCC, but has no advantage in terms of tolerability [3]. There is, therefore, an unmet need for effective and well-tolerated treatments in advanced HCC. Selective internal radiation therapy (SIRT) may provide an additional alternative for patients with unresectable HCC without extrahepatic metastases for whom curative treatments or transarterial chemoembolization (TACE) are not appropriate options, or who have failed on these treatments.

Two multicenter randomized controlled trials (RCT) in Europe (SARAH) [5] and Asia Pacific (SIRveNIB) [6] compared SIRT using ${ }^{90}$ yttrium $\left({ }^{90} \mathrm{Y}\right)$ resin microspheres (SIR-Spheres ${ }^{\circledR}$; Sirtex, Sydney, Australia) and sorafenib $400 \mathrm{mg}$ twice daily in patients with unresectable HCC not eligible for TACE. These RCTs did not show a statistically significant benefit of SIRT over sorafenib in terms of OS, but did show some benefits of SIRT in other

Future Medicine 
measures such as tumor response rate, disease progression in the liver as first event, and frequency and severity of treatment-related AEs [5,6].

The SARAH trial, which was initiated in 2009, recruited patients with HCC who were ineligible for curative treatment or had failure after up to two rounds of TACE. The recruited population had a broad range of baseline characteristics, including factors that confer a poor prognosis (such as a compromised liver function [Child-Pugh up to B7, bilirubin up to $50 \mathrm{mmol} / \mathrm{l}$ ] and/or a severe burden of disease [tumor burden $>25 \%$ of the liver, complete occlusion of the main portal vein]). These selection criteria may have resulted in the recruitment of patients who were poor candidates for SIRT $[7,8]$.

While the current European Association for the Study of the Liver (EASL) and European Society for Medical Oncology (ESMO) guidelines highlight the importance of liver function and tumor burden when selecting treatments for patients with HCC, there is no consensus on the criteria for stratifying patients to receive either locoregional therapy or systemic treatment $[9,10]$.

This post hoc subgroup analysis of the SARAH trial was, therefore, conducted to determine whether a tumor burden $\leq 25 \%$ of the total liver volume and well-preserved liver function defined as an albumin-bilrubin (ALBI) grade of 1 may act as stratification criteria to assign patients to receive either SIRT using ${ }^{90} \mathrm{Y}$ resin microspheres or sorafenib $400 \mathrm{mg}$ twice daily.

\section{Materials \& methods}

SARAH was a multicenter RCT performed at 25 centers in France (ClinicalTrials.gov, number NCT01482442) [5]. SARAH was performed in accordance with the Declaration of Helsinki, approved by an ethics committee, and complied with the provisions of the International Council for Harmonisation of Technical Requirements for Pharmaceuticals for Human Use Guidelines for Good Clinical Practice. All patients provided written informed consent [5].

The primary objective of this analysis was to explore OS in patients with tumor burden $\leq 25 \%$ and an ALBI grade of 1 in the intention-to-treat (ITT) population of the SARAH trial to inform patient selection for SIRT using ${ }^{90} \mathrm{Y}$ resin microspheres in unresectable HCC, either locally advanced, recurrent or refractory to TACE.

\section{Patients}

The inclusion criteria for SARAH have been described previously [5,11]. The main criteria were, a diagnosis of HCC, either locally advanced HCC (Barcelona Clinic Liver Cancer [BCLC] stage C) without extrahepatic metastases, recurrent $\mathrm{HCC}$ after previous surgical or thermoablative therapy with curative intent and which is ineligible for resection, transplantation or ablation, or HCC after failing up to two rounds of TACE [11].

This analysis was conducted on patients from the ITT population in the SARAH trial who had a tumor burden $\leq 25 \%$ (i.e., tumoral replacement of up to $25 \%$ of the liver volume) and an ALBI grade of 1 at baseline [5]. The ALBI grade is calculated from serum albumin and bilirubin levels based on the formula $\left(\log _{10}\right.$ bilirubin $\left.\times 0.66\right)$ + (albumin $\times-0.085)$ and categorized into three different grades: Grade $3(>-1.39)$, Grade $2(>-2.60$ to $\leq-1.39)$ and Grade $1(\leq-2.60)$, with Grade 1 reflecting better preserved liver function than Grade 2 or $3[12,13]$.

\section{Study treatment}

Eligible patients were randomly assigned (1:1) to receive either SIRT or sorafenib [5]. Oral treatment with sorafenib started at $400 \mathrm{mg}$ twice daily in the week following randomization. Treatment discontinuation or dose reduction were described in the main publication [5]. Patients assigned to SIRT underwent angiography of the hepatic artery and protective coiling of extrahepatic branches as required. Technetium- $99 \mathrm{~m}$ macroaggregated albumin ${ }^{99} \mathrm{~m} \mathrm{Tc}-$ MAA) was injected into the hepatic artery using the same catheter position chosen for the scheduled SIRT session for the liver perfusion scan. Dose activity was calculated according to the modified body surface area model [14] by the center performing SIRT [5].

Patients underwent SIRT 1 or 2 weeks after hepatic angiography using ${ }^{90}$ Y-labeled resin microspheres (SIRSpheres; Sirtex). A lobar, sectoral or segmental approach was utilized according to tumor size and location. For bilobar tumors, the first treatment was performed in the hemiliver with the greatest tumor burden, with treatment of the contralateral hemiliver scheduled 30-60 days later. SIRT could be repeated if the tumor progressed [5]. 


\section{Outcome measures}

The primary end point of interest was OS defined as the time from the date of randomization to death from any cause, or censored at the date of the last follow-up if the patient was alive.

Secondary end points were progression-free survival (PFS) defined as the time from randomization to disease progression, and the number of patients who underwent subsequent treatment with curative intent (i.e., surgical resection, liver transplantation, tumor ablation).

\section{Statistical analysis}

Analyses were performed in subgroups of the ITT population. OS and PFS were estimated using Kaplan-Meier curves. A Cox model was used to calculate hazard ratios (HR) and their two-sided 95\% CIs. A per-protocol analysis was not deemed necessary as the $92 \%$ of the patients who had been randomized to SIRT received the allocated treatment. Subgroup effects were tested using a Cox proportional hazards model incorporating an interaction term. A treatment effect favoring SIRT over sorafenib was suggested if $\mathrm{HR}<1$.

\section{Results}

Between December 2011 and March 2015, 467 patients were recruited to the SARAH trial [5], of these, eight patients subsequently withdrew consent, and 459 were included in the ITT population. It was not possible to estimate the ALBI grade for 15 patients (four in the sorafenib arm and 11 in the SIRT arm), and of these, ten had a tumor burden $\leq 25 \%$ and were excluded as they could not be assigned to the analysis group, and five had a tumor burden more than $25 \%$ and were included as they could be assigned to the population with ALBI grade $>1$ and/or tumor burden more than $25 \%$. The final study population comprised 449 patients. In this study population, 85 patients - $37(16 \%)$ patients in the SIRT arm and $48(22 \%)$ in the sorafenib arm - had a tumor burden $\leq 25 \%$ and an ALBI grade of 1 and were included in this analysis.

The number of patients in each subgroup and their baseline characteristics are reported in Table 1. Baseline characteristics were well-balanced between the treatment arms with the exception of: ECOG performance status 1, which was more frequent in the SIRT arm; and bilobar disease, which was more frequent in the SIRT arm, however, neither difference was statistically significant (Table 1 ).

Of the 37 patients in the subgroup with a tumor burden $\leq 25 \%$ and an ALBI grade 1 randomized to receive SIRT, 34 (92\%) received the treatment: all 48 patients randomized to sorafenib received the allocated treatment. In contrast, in the SARAH trial overall 53/237 (22\%) randomized to SIRT did not receive their allocated treatment; 216/222 (97\%) of patients randomized to sorafenib received their allocated treatment [5].

Median OS in the SIRT arm was 21.9 months (95\% CI: 15.2-32.5) and 17.0 months (95\% CI: 11.6-20.8) in the sorafenib arm (HR: 0.73; 95\% CI: 0.44-1.21; $\mathrm{p}=0.22$; Figure 1). The interaction effect estimate from the Cox regression was 0.609 (95\% CI: 0.344-1.079; $\mathrm{p}=0.089)$ indicating that there was a trend favoring SIRT over sorafenib in this subgroup, but statistical significance was not achieved. The effect of tumor burden and ALBI grade on the relative effectiveness of SIRT and sorafenib was also apparent when comparing with outcomes for patients with an ALBI grade $>1$ and/or a tumor burden more than 25\% (Supplementary Table 1). Median OS for these patients, who were not part of the target subgroup, was 6.5 months (95\% CI: 5.7-8.3) in the SIRT arm and 9.5 months (95\% CI: 7.1-10.3) in the sorafenib arm (Supplementary Figure 1).

A similar benefit of SIRT was observed in PFS: median PFS in the SIRT arm was 6.7 months (95\% CI: 3.9-9.5) and 3.7 months (95\% CI: 3.2-6.0) in the sorafenib arm (HR: 0.65; 95\% CI: 0.41-1.02; p = 0.06; Figure 2), but statistical significance was not achieved. Median PFS for patients with an ALBI grade $>1$ and/or a tumor burden more than 25\% was similar in the two groups; 3.9 months (95\% CI: 3.7-4.5) in the SIRT arm and 3.8 months (95\% CI: 3.3-5.6) in the sorafenib arm (Supplementary Figure 2).

A higher proportion of patients in the SIRT arm of the good liver function/low tumor burden subgroup subsequently received curative treatment than in the sorafenib arm: $14 \%(5 / 37)$ versus 2.1\% (1/48; Table 2). Patients received their subsequent curative therapy after a mean 15.8 (SD: 8.98 months; median: 10 months, range: 6-34 months) months after randomization and all were alive at a median follow-up of 25.0 months (range: 16.0-32.3). In the SARAH trial overall, $5.1 \%$ of patients who received SIRT subsequently received potentially curative treatment: all but one were alive at a median follow-up of 27.9 months [5,15]. In the sorafenib group, $1.4 \%$ of patients received potentially curative treatments [5]. 


\begin{tabular}{|c|c|c|c|}
\hline Baseline characteristic & $\operatorname{SIRT}(n=37)$ & Sorafenib $(n=48)$ & p-value \\
\hline \multicolumn{4}{|l|}{ Age (years) } \\
\hline$\geq 65$ & $16(43)$ & $23(48)$ & 0.83 \\
\hline$<65$ & $21(57)$ & $25(52)$ & \\
\hline \multicolumn{4}{|c|}{ ECOG performance status } \\
\hline 0 & $22(62)$ & $38(79)$ & 0.14 \\
\hline 1 & $15(38)$ & $10(21)$ & \\
\hline 2 & 0 & 0 & \\
\hline \multicolumn{4}{|l|}{ BCLC stage } \\
\hline A & $1(3)$ & $3(6)$ & 0.62 \\
\hline B & $16(43)$ & $17(35)$ & \\
\hline C & $20(54)$ & $28(58)$ & \\
\hline \multicolumn{4}{|c|}{ Number of tumor nodules (multifocal vs single nodule) } \\
\hline Multiple & $21(57)$ & $32(67)$ & 0.48 \\
\hline Single & $16(43)$ & $16(33)$ & \\
\hline \multicolumn{4}{|c|}{ Number of tumor nodules } \\
\hline$<4$ & $34(92)$ & $44(92)$ & 1.00 \\
\hline$\geq 4$ & $3(8)$ & $4(8)$ & \\
\hline \multicolumn{4}{|c|}{ Macroscopic vascular invasion } \\
\hline No & $17(46)$ & $23(48)$ & 1.00 \\
\hline Yes & $20(54)$ & $25(52)$ & \\
\hline \multicolumn{4}{|l|}{ Child-Pugh grade } \\
\hline A & $35(95)$ & $47(98)$ & 0.82 \\
\hline B & $2(5)$ & $1(2)$ & \\
\hline \multicolumn{4}{|l|}{ Previous TACE } \\
\hline No & $18(49)$ & $27(56)$ & 0.63 \\
\hline Yes & $19(51)$ & $21(44)$ & \\
\hline \multicolumn{4}{|c|}{ Occlusion of main portal vein } \\
\hline Absent & $33(89)$ & $43(90)$ & 1.00 \\
\hline Present & $4(11)$ & $5(10)$ & \\
\hline \multicolumn{4}{|l|}{ Liver involvement } \\
\hline Bilobar & $9(24)$ & $7(15)$ & 0.39 \\
\hline Unilobar & $28(76)$ & $41(85)$ & \\
\hline
\end{tabular}

Table 2. Subsequent potentially curative treatment in patients with $\leq 25 \%$ tumor burden and albumin-bilirubin grade 1 .

\begin{tabular}{|c|c|c|c|c|c|c|}
\hline \multirow[t]{2}{*}{ Treatment } & \multirow[t]{2}{*}{ Patient's status EOS } & \multirow[t]{2}{*}{ Follow-up (months) } & \multirow{2}{*}{$\begin{array}{l}\text { Tumor burden at } \\
\text { baseline (\%) }\end{array}$} & \multicolumn{3}{|c|}{ Potentially curative treatment received } \\
\hline & & & & $\begin{array}{l}\text { Radiofrequency } \\
\text { ablation }\end{array}$ & Surgery & Transplant \\
\hline \multirow[t]{3}{*}{ SIRT } & Alive & 25 & 4 & No & No & Yes \\
\hline & Alive & 35 & 10 & No & No & Yes \\
\hline & Alive & 33 & 6 & No & Yes & No \\
\hline
\end{tabular}




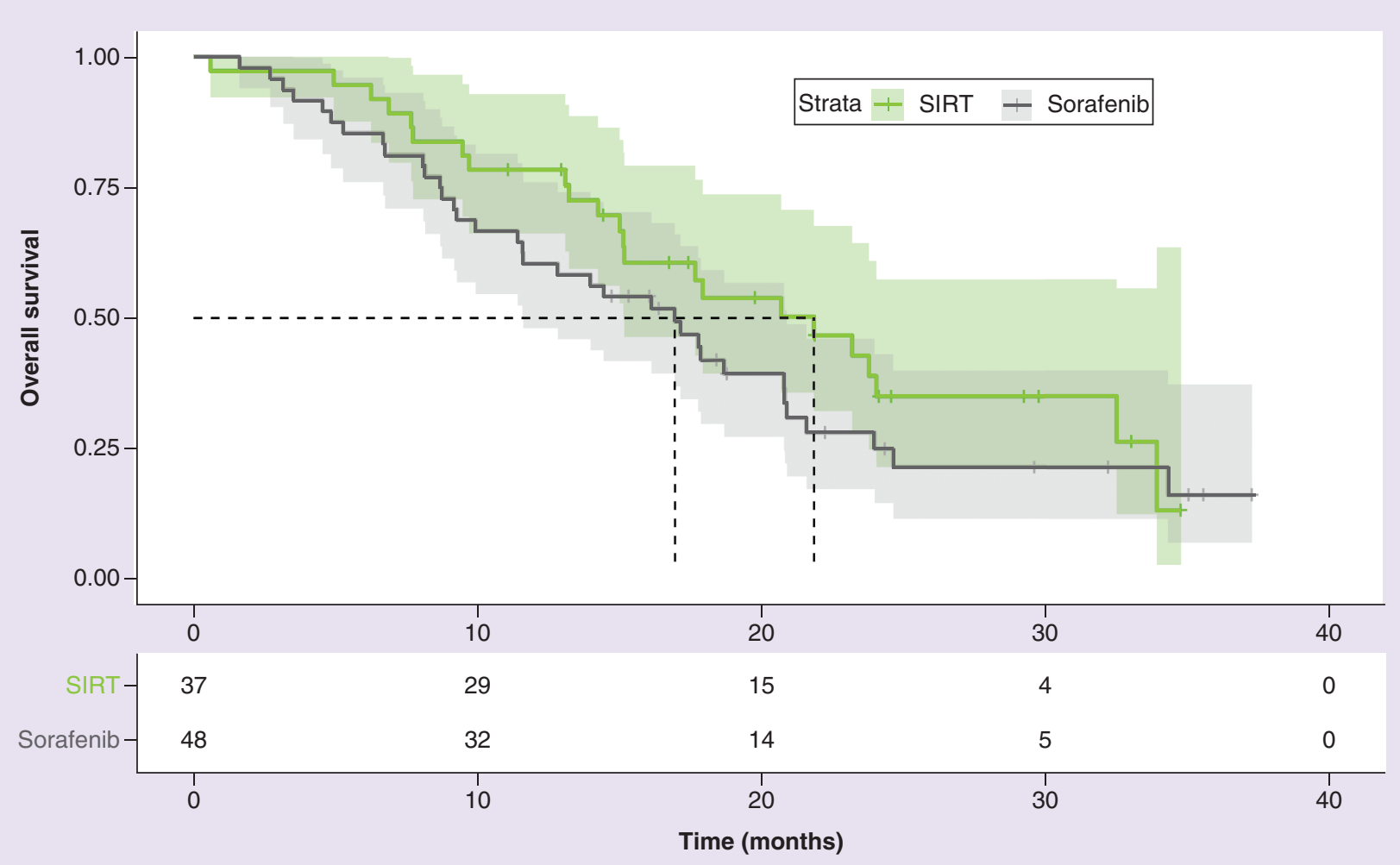

Figure 1. Kaplan-Meier plot of overall survival for selective internal radiation therapy versus sorafenib in patients with $\leq \mathbf{2 5} \%$ tumor burden and albumin-bilirubin grade 1 in the SARAH trial.

SIRT: Selective internal radiation therapy.

Safety

Among patients with an ALBI grade 1 and a tumor burden $\leq 25 \%, 24 / 37$ (65\%) patients in the SIRT arm versus $47 / 48(98 \%)$ patients in the sorafenib arm experienced at least one treatment-related $\mathrm{AE}(\mathrm{p}=0.0002)$. A statistically significant reduction in the incidence of grade $\geq 3 \mathrm{AEs}$ was also observed in the SIRT arm compared with the sorafenib arm, with respectively $9 / 37(24 \%)$ versus $30 / 48(63 \%)$ reporting at least one grade $\geq 3 \mathrm{AE}(\mathrm{p}=0.001$; Table 3). Most grade $\geq 3$ AEs were reported more frequently in the sorafenib arm than in the SIRT arm (Table 3). AEs known to be associated with sorafenib treatment, which included grade $\geq 3$ hand-foot skin reaction, were reported in $4 / 48(8.3 \%)$ of patients in the sorafenib arm and none in the SIRT arm. Diarrhea was reported in $7 / 48(15 \%)$ of patients in the sorafenib arm and none in the SIRT arm.

\section{Discussion}

This analysis of data from the SARAH trial suggests that a low tumor burden $(\leq 25 \%)$ in combination with good liver function (ALBI grade of 1) may be useful in identifying HCC patients for whom SIRT with ${ }^{90} \mathrm{Y}$ resin microspheres is associated with better OS than sorafenib. However, the HR for the OS did not reach statistical significance, possibly due to the small size of the subgroup.

In this subgroup analysis, median OS was longer in both the SIRT and sorafenib arms than in the primary analysis of the ITT population in the SARAH trial where median OS was 8.0 and 9.9 months, respectively [5]. Likewise in this subgroup, median PFS in the SIRT arm was longer than in the primary analysis of the ITT population in the SARAH trial, 6.7 and 4.1 months, respectively, while PFS in the sorafenib arm was 3.7 months both in this subgroup analysis and in the primary analysis of the SARAH study [5].

ECOG performance status 1 and bilobar disease were more frequent in the SIRT arm of this subgroup. Both of these characteristics are negative prognostic criteria. ECOG performance status of 1 is known to be a predictor of poor survival after both SIRT with ${ }^{90} \mathrm{Y}$ resin microspheres and sorafenib treatment $[16,17]$. Bilobar liver involvement is a predictor of poor survival after SIRT [17]. Consequently, baseline characteristics in this subgroup were not 


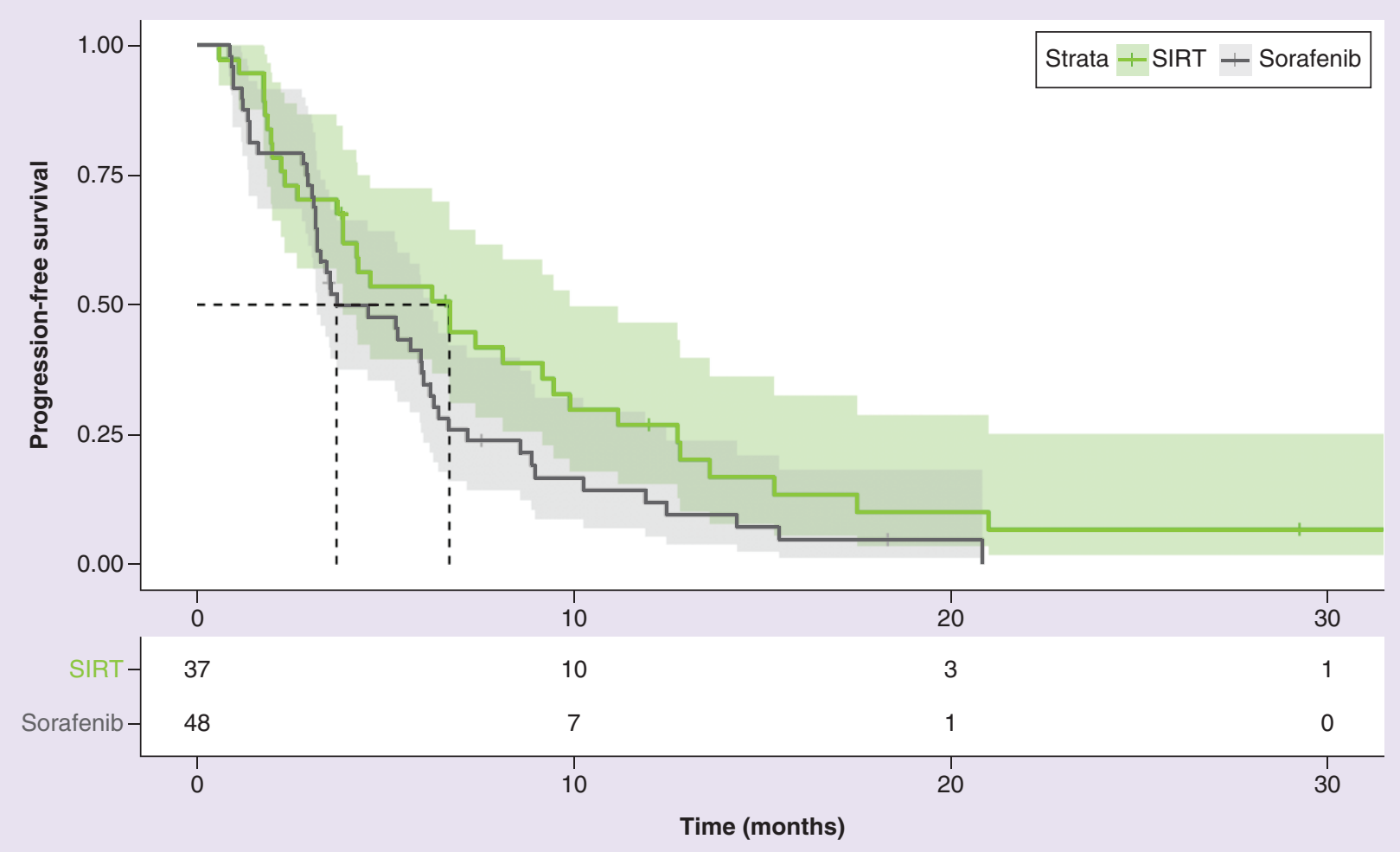

Figure 2. Kaplan-Meier plot of progression-free survival for selective internal radiation therapy versus sorafenib in patients with $\leq \mathbf{2 5} \%$ tumor burden and albumin-bilirubin grade 1 in the SARAH trial.

SIRT: Selective internal radiation therapy.

expected to favor SIRT and could result in conservative estimates of OS and PFS compared with sorafenib. Furthermore, a proportion of patients in the current analysis had previously failed TACE. The impact that previous TACE failure may have on outcomes with SIRT versus sorafenib are not known, and further research may also elucidate this as a potential criterion for patient selection.

The current ESMO and EASL guidelines acknowledge that there is no consensus on tumor volume and liver function criteria that may help in treatment selection for patients with advanced HCC, and consequently any chosen cutoffs are not fully evidence-based. The rationale for choosing a subgroup from the SARAH trial with $\leq 25 \%$ of the total liver volume was that patients with a lower tumor volume may be most likely to benefit from SIRT due to improved targeting of the liver tumors. This cutoff was predefined in the SARAH trial, and has been used for SIRT patient selection in indications other than HCC [18-25]. In addition, a cutoff of tumor burden $\leq 25 \%$ is used in some countries as a criteria for using or reimbursing SIRT in other indications [26,27]. Patients with a lower tumor burden may have received a higher dose of radiation to the tumor in the SARAH trial, especially with the use of the body surface area model for activity calculation: a tumor-absorbed dose $\geq 100$ Gy has been identified as a predictor of prolonged OS following SIRT using ${ }^{90} \mathrm{Y}$ resin microspheres [22]. It is of course true that the selection of patients with a good prognosis based on tumor burden and liver functional reserve is also likely to improve outcomes of systemic therapy with sorafenib (as is observed in this analysis when the OS in this subgroup is compared with the SARAH population overall). However, we hypothesized that the relative benefits with SIRT over sorafenib may be greater due to the differences in mode of action between SIRT and sorafenib, and their relationship to the physiological disease characteristics of HCC. Observational studies have reported a correlation between number of tumors, their maximum size and their dissemination in the liver with OS following SIRT [17,28,29], whereas the number of tumors or their maximum size were not found to be treatment effect modifiers in Phase III trials of sorafenib versus placebo [30].

The relationship between good functional reserve of the liver and reduced toxicity of SIRT to normal liver parenchyma is well-documented [24]. Sorafenib has also been shown to be less effective in patients with impaired 
Table 3. Patients experiencing a grade $\geq 3$ adverse event in the population with $\leq 25 \%$ tumor burden and albumin-bilirubin grade 1.

\begin{tabular}{|c|c|c|c|}
\hline \multirow[t]{2}{*}{ Adverse event } & \multicolumn{3}{|c|}{ Patients experiencing treatment-related adverse events } \\
\hline & SIRT $(n=37)$ & Sorafenib $(n=48)$ & p-value \\
\hline Patients with any adverse event (any grade) & $24(64.9)$ & $47(97.9)$ & 0.0002 \\
\hline - Patients with any Grade 3-4 adverse event & $9(24.3)$ & $30(62.5)$ & 0.001 \\
\hline - Abdominal pain & $1(2.7)[1]$ & $3(6.3)[3]$ & 0.803 \\
\hline - Anorexia & 0 & $3(6.3)[3]$ & 0.339 \\
\hline - Cardiac failure, congestive & 0 & $2(4.2)[2]$ & 0.593 \\
\hline - Diarrhea & 0 & 7 (14.6) [9] & 0.043 \\
\hline - Dry skin & 0 & $2(4.2)[2]$ & 0.593 \\
\hline - Fatigue & $1(2.7)[1]$ & $7(14.6)[7]$ & 0.137 \\
\hline - Gastrointestinal bleeding & $1(2.7)[1]$ & 0 & 0.896 \\
\hline - Gastrointestinal ulceration & $1(2.7)[2]$ & 0 & 0.896 \\
\hline - Hemorrhage (nongastrointestinal) & 0 & $2(4.2)[2]$ & 0.593 \\
\hline - Hand-foot skin reaction & 0 & $4(8.3)[5]$ & 0.200 \\
\hline - Hyperbilirubinemia & 0 & $1(2.1)[1]$ & 1.000 \\
\hline - Infection & 0 & $1(2.1)[3]$ & 1.000 \\
\hline - Liver dysfunction & 0 & $3(6.3)[3]$ & 0.339 \\
\hline - Nausea or vomiting & 0 & $1(2.1)[1]$ & 1.000 \\
\hline - Other dermatological events & 0 & $1(2.1)[1]$ & 1.000 \\
\hline - Other increased liver values & $1(2.7)[1]$ & $1(2.1)[1]$ & 1.000 \\
\hline - Renal dysfunction (increased creatinine) & $1(2.7)[1]$ & $1(2.1)[1]$ & 1.000 \\
\hline
\end{tabular}

liver function (Child-Pugh B) [31,32]. As ALBI grade provides an objective estimate of hepatic functional reserve [33], we used the cutoff of ALBI grade 1 as a definition of well-preserved liver function. The ALBI grade is recognized as a valid criterion for stratification of patients in prognostic groups of liver function within the Child-Pugh A class [9,10,34], especially for patients with HCC receiving locoregional therapy $[17,28,29,35]$ as ALBI considered a better estimator of the liver functional reserve than Child-Pugh [10]. It was hypothesized that patients with well-preserved liver function may be most likely to benefit from SIRT, because baseline serum albumin and bilirubin have previously been identified as predictors of survival and toxicity outcomes of SIRT using ${ }^{90} \mathrm{Y}$ resin microspheres [12,13,17,24,28,29,34-37].

Outcomes of this analysis support the concept that the selection of patients with an ALBI grade 1 and a tumor burden $\leq 25 \%$ is more influential on the outcomes of SIRT than it is for sorafenib. This is most apparent when comparing PFS outcomes for the target subgroup versus the rest of the ITT population (i.e., ALBI grade $>1$ and/or tumor burden $>25 \%$ ): in the SIRT arm, median PFS was 6.7 months in the subgroup in contrast to 3.9 months in the rest of the ITT population; in the sorafenib arm, median PFS was 3.7 months in the subgroup and 3.8 months in the rest of the ITT population.

A proportion of patients who receive SIRT for HCC that is unresectable at presentation may be downstaged, in other words, may have a significant tumor response following therapy to become eligible for potentially curative treatments $[10,24]$. In this subgroup analysis, a markedly higher proportion of patients who received SIRT were downstaged to receive curative treatment than those treated with sorafenib. Patients are expected to have longerterm survival after curative treatment [38,39], with a recent study showing higher OS for patients downstaged following SIRT and even higher for those who received subsequent curative treatment [40]. However, due to the censoring of the data in the SARAH trial, the survival benefits of downstaging to curative treatment following SIRT, compared with sorafenib, may not have been observed in survival analyses in the trial.

AEs associated with sorafenib are frequent and may lead to the discontinuation of treatment $[1,2,4]$. In this subgroup analysis, the incidence of any treatment-related AEs and grade $\geq 3$ AEs was lower in the SIRT arm than in the sorafenib arm, and the safety benefit of SIRT over sorafenib in this subgroup was greater than in the overall 
ITT population. Furthermore, AEs known to have an adverse effect on patients' quality of life, such as grade $\geq 3$ hand-foot skin reactions and diarrhea, predominantly occurred among patients who received sorafenib.

There are limitations to this analysis. Low tumor burden $(\leq 25 \%)$ and good liver function was not a prespecified subgroup in the SARAH trial. The ALBI score was not reported in the SARAH trial, but serum albumin and bilirubin level data were collected. There are other potential prognostic factors for the stratification of HCC patients to SIRT or systemic treatment. Adding other factors, such as BCLC stage or presence of macroscopic vascular invasion, to the analysis may have resulted in a numerically larger benefit of SIRT versus sorafenib, and thus may have given further guidance on patient selection, but would have further reduced the sample sizes and prevented meaningful interpretation. Furthermore, univariate analyses of OS stratified by BCLC stage or macroscopic vascular invasion did not demonstrate a benefit of SIRT using ${ }^{90} \mathrm{Y}$ resin microspheres in the ITT and per protocol populations of the SARAH trial [5].

A pooled analysis of data from both the SARAH and SIRveNIB studies, to increase the number of patients available for analysis, may have provided strength to the findings, but this was not feasible. The SIRveNIB study employed a different tumor burden cutoff and individual values of tumor burden were not available for the sorafenib arm. Furthermore, baseline characteristics, risk factors for HCC and patient recruitment in the SIRveNIB trial differed from those in SARAH, with the distribution of risk factors for HCC in SIRveNIB reflecting those in Asia-Pacific population rather than a western population [5,6,10,41]. Hence, we focused this analysis on data from the SARAH trial.

\section{Conclusion}

This analysis of the SARAH data supports the proposition that the combination of good liver function (ALBI grade 1$)$ and low tumor burden $(\leq 25 \%)$ may be relevant in the selection of patients with locally advanced HCC for SIRT with ${ }^{90} \mathrm{Y}$ resin microspheres. This hypothesis warrants further research.

\section{Summary points}

- SARAH, a multicenter randomized controlled trial comparing selective internal radiation therapy (SIRT) using ${ }^{90}$ yttrium $\left({ }^{90} \mathrm{Y}\right)$ resin microspheres and sorafenib $400 \mathrm{mg}$ twice daily in patients with unresectable hepatocellular carcinoma (HCC) not eligible for transarterial chemoembolization did not show a statistically significant benefit of SIRT over sorafenib in terms of overall survival.

- SARAH showed some benefits of SIRT in other measures such as tumor response rate, and frequency and severity of treatment-related adverse events (AEs).

- In SARAH, the recruited population had a broad range of baseline characteristics, including factors that confer a poor prognosis (e.g., compromised liver function and/or a severe tumor burden [ $>25 \%$ of the liver]) that may have led to the enrollment of patients who were poor candidates for SIRT.

- This post-hoc subgroup analysis of SARAH aimed to determine whether a liver tumor burden $\leq 25 \%$ and well-preserved liver function (albumin-bilrubin grade 1) are appropriate criteria for identifying patients with advanced HCC who may benefit from SIRT using ${ }^{90} \mathrm{Y}$ resin microspheres versus sorafenib.

- There was a trend for improved median overall survival in the SIRT arm (21.9 months; $95 \% \mathrm{Cl}: 15.2-32.5, \mathrm{n}=37)$ compared with the sorafenib arm (17.0 months; $95 \% \mathrm{Cl}: 11.6-20.8, \mathrm{n}=48$; hazard ratio: $0.73 ; 95 \% \mathrm{Cl}$ : $0.44-1.21 ; p=0.22$ ).

- Median progression-free survival was longer in the SIRT arm (6.7 months; 95\% Cl: 3.9-9.5) and than in the sorafenib arm (3.7 months; $95 \% \mathrm{Cl}$ : 3.2-9.5; hazard ratios: $0.65 ; 95 \% \mathrm{Cl}: 0.41-1.02 ; \mathrm{p}=0.06$ ).

- Incidence of any treatment-related AEs and grade $\geq 3$ AEs was lower in the SIRT arm than in the sorafenib arm, in this analysis.

- This analysis of SARAH data supports the proposition that the combination of good liver function (albumin-bilrubin grade 1 ) and low tumor burden $(\leq 25 \%)$ may be relevant in the selection of patients with locally advanced HCC for SIRT with ${ }^{90} \mathrm{Y}$ resin microspheres.

Supplementary data

To view the supplementary data that accompany this paper please visit the journal website at: www.futuremedicine.com/doi/sup $\mathrm{pl} / 10.2217 /$ fon-2019-0658

Author contributions

DH Palmer developed the concept of the study. DH Palmer and PJ Ross were responsible for the design and conduct of the study. NS Hawkins designed and conducted the statistical analyses. V Vilgrain was the principal investigator of the SARAH trial. V Vilgrain, H 
Pereira and G Chatellier provided data for the study and reviewed the statistical analyses. All the authors contributed to the writing, critical review of the manuscript for scientific content and accuracy, and gave final approval for submission of the manuscript.

\section{Acknowledgments}

The authors acknowledge the SARAH trial group, who conducted the original trial, and their support in the preparation of this paper.

Financial \& competing interests disclosure

The authors indicated that financial assistance was provided by Sirtex Technology Ltd. DH Palmer is supported by the Liverpool CR UK/NIHR Experimental Cancer Medicine Centre and reports fees/honoraria/travel support from Bayer, Eisai, BMS, MSD and AstraZeneca. NS Hawkins is a partner for Visible Analytics Ltd., which conducted the statistical analyses and received consultancy, research grant and expenses from Sirtex. PJ Ross reports honoraria from Amgen, Servier, Shire, Sirtex, BMS, Pierre Fabre and Roche; participation in advisory board meetings for Shire, Celgene, BMS, Pierre Fabre; receipt of travel support from Amgen, BMS, Bayer, Roche and Servier; his institution has received research funding from Sanofi. V Vilgrain reports research grants and personal fees from Sirtex during the conduct of the SARAH study, and subsequent participation in an advisory board meeting for Sirtex. G Chatellier and $\mathrm{H}$ Pereira declare no competing interests. The authors have no other relevant affiliations or financial involvement with any organization or entity with a financial interest in or financial conflict with the subject matter or materials discussed in the manuscript apart from those disclosed.

Writing and editorial support was provided by Martin Gilmour and Tim Latham of ESP Bioscience Ltd., funded by Sirtex Medical Ltd.

\section{Ethical conduct of research}

The authors state that SARAH was performed in accordance with the Declaration of Helsinki, approved by an ethics committee, and complied with the provisions of the International Council for Harmonisation of Technical Requirements for Pharmaceuticals for Human Use Guidelines for Good Clinical Practice (ICH-GCP). All patients provided written informed consent.

Open access

This work is licensed under the Attribution-NonCommercial-NoDerivatives 4.0 Unported License. To view a copy of this license, visit http://creativecommons.org/licenses/by-nc-nd/4.0/

\section{Data sharing statement}

The authors certify that this manuscript reports the secondary analysis of clinical trial data that have been shared with them, and that the use of this shared data is in accordance with the terms (if any) agreed upon their receipt. The source of this data is: SARAH trial; NCT01482442

\section{References}

Papers of special note have been highlighted as: $\bullet$ of interest; $\bullet \bullet$ of considerable interest

1. Cheng AL, Kang YK, Chen Z et al. Efficacy and safety of sorafenib in patients in the Asia-Pacific region with advanced hepatocellular carcinoma: a Phase III randomised, double-blind, placebo-controlled trial. Lancet Oncol. 10(1), 25-34 (2009).

-. This is a key Phase III study supporting the use of sorafenib in the treatment of advanced hepatocellular carcinoma (HCC).

2. Llovet JM, Ricci S, Mazzaferro V et al. Sorafenib in advanced hepatocellular carcinoma. N. Engl. J. Med. 359(4), 378-390 (2008).

-. This is a key Phase III study supporting use of sorafenib in the treatment of advanced HCC.

3. Kudo M, Finn RS, Qin S et al. Lenvatinib versus sorafenib in first-line treatment of patients with unresectable hepatocellular carcinoma: a randomised Phase III non-inferiority trial. Lancet 391(10126), 1163-1173 (2018).

4. Pinter M, Sieghart W, Graziadei I et al. Sorafenib in unresectable hepatocellular carcinoma from mild to advanced stage liver cirrhosis. Oncologist 14(1), 70-76 (2009).

5. Vilgrain V, Pereira $\mathrm{H}$, Assenat $\mathrm{E}$ et al. Efficacy and safety of selective internal radiotherapy with yttrium-90 resin microspheres compared with sorafenib in locally advanced and inoperable hepatocellular carcinoma (SARAH): an open-label randomised controlled Phase III trial. Lancet Oncol. 18(12), 1624-1636 (2017).

-. This is the full publication of the SARAH study.

6. Chow PKH, Gandhi M, Tan SB et al. SIRveNIB: selective internal radiation therapy versus sorafenib in Asia-Pacific patients with hepatocellular carcinoma. J. Clin. Oncol. 36(19), 1913-1921 (2018).

-. This is a key Phase III study comparing selective internal radiation therapy using ${ }^{90} \mathrm{Y}$ resin microsphere with sorafenib in the treatment of HCC. 
7. Garin E, Rolland Y, Campillo-Gimenez B, Edeline J. Negative Phase III study of (90)Y microspheres versus sorafenib in HCC. Lancet Oncol. 19(2), e70 (2018).

8. Kokudo T, Kokudo N, Hasegawa K. Negative Phase III study of (90)Y microspheres versus sorafenib in HCC. Lancet Oncol. 19(2), e68 (2018).

9. European Association for the Study of the Liver. EASL Clinical Practice Guidelines: management of hepatocellular carcinoma. J. Hepatol. 69(1), 182-236 (2018).

- Current guidelines on the management of HCC.

10. Vogel A, Cervantes A, Chau I et al. Hepatocellular carcinoma: ESMO Clinical Practice Guidelines for diagnosis, treatment and follow-up. Ann. Oncol. 29(Suppl. 4), iv238-iv255 (2018).

- Current guidelines on the management of HCC.

11. Bruix J, Sherman M. . Management of hepatocellular carcinoma: an update. Hepatology 53(3), 1020-1022 (2011).

12. Hiraoka A, Kumada T, Tsuji $\mathrm{K}$ et al. Validation of modified ALBI grade for more detailed assessment of hepatic function in hepatocellular carcinoma patients: a multicenter analysis. Liver Cancer 8(2), 121-129 (2019).

13. Johnson PJ, Berhane S, Kagebayashi C et al. Assessment of liver function in patients with hepatocellular carcinoma: a new evidence-based approach-the ALBI grade. J. Clin. Oncol. 33(6), 550-558 (2015).

14. Lau W, Kennedy A, Kim Y et al. Patient selection and activity planning guide for selective internal radiotherapy with yttrium-90 resin microspheres. Int. J. Radiat. Oncol. Biol. Phys. 82(1), 401-407 (2012).

15. Sirtex. Data on File. (2019).

16. King J, Palmer DH, Johnson P et al. Sorafenib for the treatment of advanced hepatocellular cancer - a UK audit. Clin. Oncol. 29(4), 256-262 (2017).

17. Sangro B, Carpanese L, Cianni R et al. Survival after yttrium-90 resin microsphere radioembolization of hepatocellular carcinoma across Barcelona clinic liver cancer stages: a European evaluation. Hepatology 54(3), 868-878 (2011).

18. White J, Carolan-Rees G, Dale M et al. Analysis of a National Programme for selective internal radiation therapy for colorectal cancer liver metastases. Clin. Oncol. 31(1), 58-66 (2019).

19. Saxena A, Bester L, Shan L et al. A systematic review on the safety and efficacy of yttrium-90 radioembolization for unresectable, chemorefractory colorectal cancer liver metastases. J. Cancer Res. Clin. Oncol. 140(4), 537-547 (2014).

20. Gebski V, Gibbs E, Gandhi M et al. VESPRO: an individual patient data prospective meta-analysis of selective internal radiation therapy versus sorafenib for advanced, locally advanced, or recurrent hepatocellular carcinoma of the SARAH and SIRveNIB trials. JMIR Res. Protoc. 6(2), e17 (2017)

21. Vilgrain V, Bouattour M, Sibert A et al. SARAH: a randomised controlled trial comparing efficacy and safety of selective internal radiation therapy (with yttrium-90 microspheres) and sorafenib in patients with locally advanced hepatocellular carcinoma. Presented at: The International Liver Congress. Amsterdam, The Netherlands (19-23 April, 2017).

22. Hermann A-L, Dieudonné A, Ronot $\mathrm{M}$ et al. Role of 99mTc-macroaggregated albumin SPECT/CT based dosimetry in predicting survival and tumor response of patients with locally advanced and inoperable hepatocellular carcinoma (HCC) treated by selective intra-arterial radiation therapy (SIRT) with yttrium-90 resin microspheres, a cohort from SARAH study. J. Hepatol. 68, S13 (2018).

23. Sancho L, Rodriguez-Fraile M, Bilbao JI et al. Is a Technetium-99m macroaggregated albumin scan essential in the Workup for selective internal radiation therapy with yttrium-90? An analysis of 532 patients. J. Vasc. Interv. Radiol. 28(11), 1536-1542 (2017).

24. Sangro B, Martinez-Urbistondo D, Bester L et al. Prevention and treatment of complications of selective internal radiation therapy: expert guidance and systematic review. Hepatology 66(3), 969-982 (2017).

25. Lau WY, Teoh YL, Win KM et al. Current role of selective internal radiation with yttrium-90 in liver tumors. Future Oncol. 12(9), 1193-1204 (2016).

26. NHS England. Clinical Commissioning Policy: selective internal radiation therapy (SIRT) for chemotherapy refractory/intolerant metastatic colorectal cancer (adults). NHS England Reference: 170102P (2018). www.england.nhs.uk/wp-content/uploads/2018/12/Se lective-internal-radiation-therapy-for-chemotherapy-refractory-intolerant-metastatic-colorectal-cancer.pdf

27. Journal Officiel de la République Française. [Order of 1 March 2019 amending the registration conditions for Yttrium-90 SIR-SPHERES microspheres from SIRTEX MEDICAL EUROPE GmbH listed in Title III of the list of reimbursable products and services provided for in Article L165-1 of the Code of Social Security] (2019). www.legifrance.gouv.fr/eli/arrete/2019/2013/2011/SSAS1906504A/jo/texte

28. Mantry PS, Mehta A, Madani B, Mejia A, Shahin I. Selective internal radiation therapy using yttrium-90 resin microspheres in patients with unresectable hepatocellular carcinoma: a retrospective study. J. Gastrointest. Oncol. 8(5), 799-807 (2017).

29. Khor AY, Toh Y, Allen JC et al. Survival and pattern of tumor progression with yttrium-90 microsphere radioembolization in predominantly hepatitis B Asian patients with hepatocellular carcinoma. Hepatol. Int. 8(3), 395-404 (2014).

30. Bruix J, Cheng AL, Meinhardt G, Nakajima K, De Sanctis Y, Llovet J. Prognostic factors and predictors of sorafenib benefit in patients with hepatocellular carcinoma: analysis of two Phase III studies. J. Hepatol. 67(5), 999-1008 (2017). 
31. Marrero JA, Kudo M, Venook AP et al. Observational registry of sorafenib use in clinical practice across Child-Pugh subgroups: the GIDEON study. J. Hepatol. 65(6), 1140-1147 (2016).

32. Amzal B, Fu S, Meng J, Lister J, Karcher H. Cabozantinib versus everolimus, nivolumab, axitinib, sorafenib and best supportive care: a network meta-analysis of progression-free survival and overall survival in second line treatment of advanced renal cell carcinoma. PLoS ONE 12(9), e0184423 (2017).

33. Hiraoka A, Michitaka K, Kumada T, Kudo M. ALBI score as a novel tool in staging and treatment planning for hepatocellular carcinoma: advantage of ALBI Grade for universal assessment of hepatic function. Liver Cancer 6(4), 377-379 (2017).

34. Hickey R, Mouli S, Kulik L et al. Independent analysis of Albumin-Bilirubin Grade in a 765-patient cohort treated with transarterial locoregional therapy for hepatocellular carcinoma. J. Vasc. Interv. Radiol. 27(6), 795-802 (2016).

35. Gil-Alzugaray B, Chopitea A, Inarrairaegui M et al. Prognostic factors and prevention of radioembolization-induced liver disease. Hepatology 57(3), 1078-1087 (2013).

36. Pinato DJ, Sharma R, Allara E et al. The ALBI grade provides objective hepatic reserve estimation across each BCLC stage of hepatocellular carcinoma. J. Hepatol. 66(2), 338-346 (2017).

37. Waked I, Berhane S, Toyoda H et al. Transarterial chemo-embolisation of hepatocellular carcinoma: impact of liver function and vascular invasion. Br. J. Cancer 116(4), 448-454 (2017).

38. Kanwal F, Befeler A, Chari RS et al. Potentially curative treatment in patients with hepatocellular cancer-results from the liver cancer research network. Aliment. Pharmacol. Ther. 36(3), 257-265 (2012).

39. Inarrairaegui M, Pardo F, Bilbao JI et al. Response to radioembolization with yttrium-90 resin microspheres may allow surgical treatment with curative intent and prolonged survival in previously unresectable hepatocellular carcinoma. Eur. J. Surg. Oncol. 38(7), 594-601 (2012).

40. Regnault HP, Perrin C, Amaddeo G et al. Yttrium-90 trans arterial radioembolisation (TARE) is an effective treatment for the downstaging of patients with hepatocellular carcinoma: a monocentric experience in a tertiary center. Presented at: EASL HCC Summit. Lisbon, Portugal (14-16 February, 2019).

41. Villanueva A. Hepatocellular carcinoma. N. Engl. J. Med. 380(15), 1450-1462 (2019). 
